\title{
Spin-polarized tunneling through a diluted magnetic semiconductor quantum dot
}

\author{
Kai Chang ${ }^{1,2, *}$ and K. S. Chan ${ }^{2}$ \\ ${ }^{1}$ NLSM, Institute of Semiconductor, Chinese Academy of Sciences, P. O. Box 912, 100083, Beijing, China \\ ${ }^{2}$ Department of Physics and Materials Science, City University of Hong Kong, Kowloon, Hong Kong, China \\ F. M. Peeters \\ Department of Physics, University of Antwerp (Campus Drie Eiken), B-2610 Antwerpen, Belgium \\ (Received 25 June 2004; revised manuscript received 17 November 2004; published 12 April 2005)
}

\begin{abstract}
Spin-polarized tunneling through a diluted magnetic semiconductor quantum dot embedded in a tunneling barrier is investigated using the Bardeen transfer Hamiltonian. The tunneling current oscillates with an increasing magnetic field for a fixed bias. Many peaks are observed with an increasing external bias under a fixed magnetic field. Spin polarization of the tunneling current is tuned by changing the external bias under a weak magnetic field.
\end{abstract}

DOI: 10.1103/PhysRevB.71.155309

PACS number(s): 73.63.Kv, 72.25.Dc, 73.21.La

\section{INTRODUCTION}

Diluted magnetic semiconductors (DMS) provide us with an interesting possibility to tailor the spin splitting and the spin polarization via the $s-d$ exchange interaction between the conduction-band electron and the localized magnetic ions. The spin splitting in DMS systems can be tuned by changing the external magnetic field, which induces spinpolarized transport. The spin-polarized current in a semiconductor is the crucial ingredient for spintronic devices. ${ }^{1}$ One of the main obstacles for these applications is to find a highly efficient way to inject spin-polarized carriers into a semiconductor. The successful spin-polarized current through a DMS junction was demonstrated theoretically ${ }^{2}$ and experimentally by applying strong magnetic fields at low temperature, ${ }^{3}$ i.e., the three-dimensional-two-dimensional-three-dimensional (3D-2D-3D) tunneling case. An oscillating, tunneling, magnetoresistance was found theoretically for ballistic DMS single-barrier and double-barrier structures ${ }^{4}$ even in the case of a superlattice. ${ }^{5}$ In all of the above studies, a strong magnetic field is required to enhance the spin splitting and to induce spin polarization of the electron. Such a strong magnetic field is a big obstacle for practical applications in spintronic devices, e.g., to create a spin filter.

Very recently, ${ }^{6-8}$ the incorporation of $\mathrm{Mn}$ ions into the crystal matrix of different II-VI semiconductors lead to the successful fabrication of DMS quantum dots (QD) and magnet-DMS hybrid structures. Photoluminescence (PL) signals clearly demonstrated the confinement of quasi-zerodimensional electron-hole pairs in these nanostructures. The optical property of the DMS QD can be well understood by calculations based on the effective-mass theory. ${ }^{9}$ In this paper, we calculate the spin-polarized tunneling current utilizing magnetotunneling through a semiconductor quantum dot and demonstrate theoretically that strong spin-polarized tunneling can be realized in this system in the presence of a weak perpendicular magnetic field. Furthermore, we find that the spin polarization of the current can be easily tuned by changing the external bias under a weak magnetic field. Thus, such a device will be very helpful for potential spintronic nanodevice applications.
The paper is organized as follows. The theoretical model and formalism are presented in Sec. II. In Sec. III we present the numerical results along with our discussions. A brief conclusion is given in Sec. IV.

\section{THEORETICAL MODEL AND FORMALISM}

The typical semiconductor structures used to study vertical tunneling through quantum dots consist of a doublebarrier quantum well that is confined in the lateral direction. The lateral confinement can be realized using ecthing, ion bombardment, or metallic gates. Consider a 2D-0D-2D structure, which is shown schematically in Fig. 1; the emitter and collector regions consist of a nonmagnetic (NMS) or diluted magnetic semiconductor two-dimensional electron gas (2DEG), and the NMS or DMS quantum dot is embedded in the tunneling barrier. The Hamiltonian of an electron in the emitter region (DMS or NMS 2DEG) is

$$
\hat{H}=\frac{\hbar^{2}}{2 m_{\|}^{*}}\left(\mathbf{k}_{\|}+e \mathbf{A}\right)^{2}+H_{Z}+\hat{H}_{\mathrm{ex}}+\frac{\hbar^{2} k_{z}^{2}}{2 m_{\perp}^{*}}+V_{\text {conf }}^{e}(z),
$$

where the magnetic field $B$ is applied along the $z$ axis, i.e., the current direction. $k_{z}$ is the momentum along the $z$ axis. We take $m_{\|}^{*}=m_{\perp}^{*}=m^{*}$, since the conduction band is isotropic near the $\Gamma$ point. The form of the confining potential $V_{\text {conf }}^{e}(z)$ is of a triangular shape which depends on the external bias,

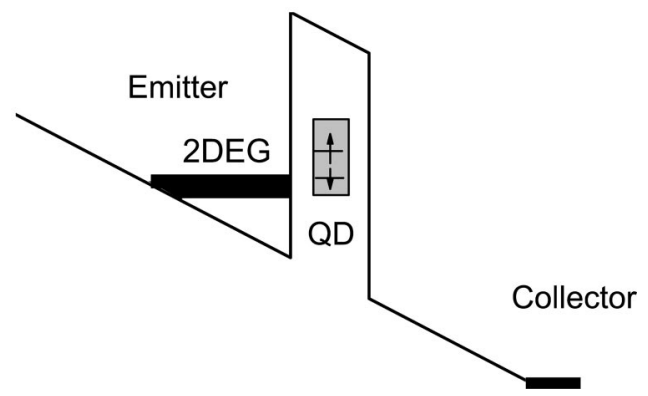

FIG. 1. Potential profile of the tunneling structure. The quantum dot is embedded in the barrier. 
and we assume that only the lowest subband $E_{0}^{e}$ of this accumulation layer is filled and contributes to the tunneling current. The symmetric gauge is adopted for the vector potential $\mathbf{A}=(-B y / 2, B x / 2,0)$ in order to optimally use the cylinder symmetry of the system. $H_{Z}$ is the intrinsic Zeeman term. $V_{c}(z)$ is the confining potential along the growth direction. The exchange interaction $H_{\mathrm{ex}}$ describes the $s-d$ interaction between the conduction electrons and the $3 d^{5}$ electrons of the Mn ions,

$$
\hat{H}_{\mathrm{ex}}=\left\{\begin{array}{ll}
0, & \text { for NMS 2DEG } \\
\sum_{i} J_{s-d}\left(\vec{r}-\vec{R}_{i}\right) \vec{\sigma} \cdot \vec{S}_{i}, & \text { for DMS 2DEG }
\end{array}\right\},
$$

where $J_{s-d}$ is the electron-ion $s-d$ exchange coupling constant, and $\vec{r}$ and $\vec{R}_{i}$ are coordinates of the band electron and the $\mathrm{Mn}^{2+}$ ions. $\vec{\sigma}$ and $\vec{S}_{i}$ denote the spin operator of the conduction-band electron and $\mathrm{Mn}^{2+}$ ion, respectively. The summation is over the lattice sites occupied by the $\mathrm{Mn}^{2+}$ ions. Within the mean-field approximation, $\vec{S}_{i}$ can be replaced by its thermal average, $\left\langle S_{z}\right\rangle=-S_{0} B_{5 / 2}(\xi), \quad \xi$ $=g_{\mathrm{Mn}} S \mu_{B} B / k_{B}\left(T+T_{0}\right) . B_{J}(x)$ is the Brillouin function, $k_{B}$ is the Boltzmann constant, $S=\frac{5}{2}$ is the spin of $\mathrm{Mn}^{2+}$, and $S_{0}$ and $T_{0}$ are phenomenological parameters describing the antiferromagnetic superexchange effect between the $\mathrm{Mn}^{2+}$ ions. Within the mean-field approximation, we have

$$
H_{\mathrm{ex}}=N_{0} \alpha x \sigma_{z}\left\langle S_{z}\right\rangle,
$$

where $N_{0}$ is the number of cation sites per unit volume, $x$ is the concentration of Mn ions, and $\alpha$ is the absolute value of the exchange-coupling integral per unit cell.

The energy of the eigenstate in the $2 \mathrm{DEG}$ is

$$
E_{n_{e}, m_{e}, k_{z}}=\hbar \omega_{c}\left(n_{e}+\frac{m_{e}+\left|m_{e}\right|}{2}+\frac{1}{2}\right)+E_{Z}^{e}+E_{Z}^{s-d}+E_{0}^{e},
$$

where $\omega_{c}=e B / m^{*}, E_{0}^{e}$ is the ground-state energy of the electron along the $z$ axis, and $E_{Z}^{e}$ and $E_{Z}^{s-d}$ denote the intrinsic Zeeman energy and the spin splitting caused by the $s-d$ exchange interaction, respectively. $E_{Z}^{s-d}$ is zero for the NMS 2DEG and nonzero for the DMS 2DEG. The wave function of the electron is

$$
\Psi_{e}(r, \varphi, z)=\frac{e^{\mathrm{im} \varphi}}{\sqrt{2 \pi}} R_{n_{e} m_{e}}(r) f(z),
$$

where $R_{n_{e} m_{e}}(r)$ is a function that can be expressed in terms of the hypergeometric function,

$$
\begin{gathered}
R_{n_{e} m_{e}}(r) \propto e^{-\xi / 2} \xi^{|m| / 2} F(-n,|m|+1, \xi), \\
\xi=\omega_{c} m^{*} r^{2} / 2 \hbar .
\end{gathered}
$$

In the QD structure, we have

$$
H=\frac{\hbar^{2} k_{\|}^{2}}{2 m^{*}}+\frac{m^{*} \omega_{0}^{2} r^{2}}{2}+\frac{L_{z} \omega_{c}}{2}+H_{Z}+H_{\mathrm{ex}}+\frac{\hbar^{2} k_{z}^{2}}{2 m}+V_{c}^{d}(z),
$$

where $\omega_{d}=\sqrt{\omega_{0}^{2}+\omega_{c}^{2} / 4}$, and $V_{c}^{d}(z)$ is the confining potential in the QD along the $z$ axis, which is assumed to be a square well. $H_{\mathrm{ex}}$ is described in Eq. (2). Assuming that the quantum confinement along the $z$ axis is much stronger than the inplane confinement, the energy of the eigenstate is

$$
E_{n_{d}, m_{d}}=\hbar \omega_{d}\left(2 n_{d}+\left|m_{d}\right|+1\right)+\frac{\hbar \omega_{c}}{2} m_{d}+E_{Z}^{e}+E_{Z}^{s-d}+E_{0}^{d} .
$$

The value of the exchange interaction term $E_{Z}^{s-d}$ depends on the material [see Eq. (4)]. The wave function is $\Psi_{d}(r, \varphi, z)$ $=1 / \sqrt{2 \pi} e^{\mathrm{im}}{ }_{d \varphi}^{\varphi} R_{n_{d} m_{d}}(r) g(z)$, where $R_{n_{d} m_{d}}(r)$ is the same as in Eq. (6), but $\xi=\omega_{d} m^{*} r^{2} / \hbar$.

The tunneling process is a two-step process in which electrons first tunnel from the emitter to the dot and eventually from the dot to the collector. At equilibrium the tunneling current from the emitter to the dot must be equal to that from the dot to the collector, and the strength of the tunneling current is determined by the overlap integral between the wave function of the QD states and those of the contacts. According to Bardeen's description of tunneling, ${ }^{10}$ the tunneling current in the steady state is given by ${ }^{11}$

$$
\begin{aligned}
I= & \frac{e}{h} \sum_{\sigma} \int d k d k^{\prime} T_{L R}\left(E_{L}, E_{R}\right)\left[1-f^{R}\left(E_{R}\right)\right] f^{L}\left(E_{L}\right) \\
& -\frac{e}{h} \sum_{\sigma} \int d k d k^{\prime} T_{R L}\left(E_{R}, E_{L}\right)\left[1-f^{L}\left(E_{L}\right)\right] f^{R}\left(E_{R}\right),
\end{aligned}
$$

where $T_{L R}\left(E_{L}, E_{R}\right)$ is the transmission of electron tunneling from the emitter to the dot and $T_{R L}\left(E_{R}, E_{L}\right)$ is the same, but for the reverse direction. The Pauli blocking factors $1-f^{L(R)}$ are introduced, considering that an electron that tunnels from one side to the other side should find an empty final state. For resonant tunneling $E_{n_{e}, m_{e}, k_{z}}=E_{n_{d}, m_{d}}, T_{L R}=T_{R L}$, and we assume that the ground state of the QD is high above the Fermi energy of the collector, which makes the Pauli blocking factor unimportant. Then the tunneling current can be obtained from

$$
I=2 e \Gamma / \pi \sum_{\sigma, n_{d}, m_{d}, n_{e}, m_{e}} \frac{\left|M_{e-d}\right|^{2} f\left(E_{n_{d}, m_{d}}\right)}{\left(E_{n_{e}, m_{e}}-E_{n_{d}, m_{d}}-e V_{\mathrm{ext}}\right)^{2}+\Gamma^{2}},
$$

where $f(E)=\left(1+\exp \left[\left(E-E_{F}\right) / k_{B} T\right]\right)^{-1}$ is the Fermi distribution function with Fermi energy $E_{F}$, which can be determined from the following equation:

$$
\aleph_{e}=e^{2} / 4 \pi l_{B}^{2} \sum_{\sigma, n_{e}, m_{e}} f\left(E_{n_{e}, m_{e}}\right),
$$

where $\aleph_{e}$ is the density of electrons in the 2DEG. In our numerical calculation the density of states (DOS) of the 2DEG, assuming a simple Lorentzian spectral function, is $\rho(E)=(\Gamma / \pi) /\left[\left(E_{n_{e}, m_{e}}-E_{n_{d}, m_{d}}-e V_{\mathrm{ext}}\right)^{2}+\Gamma^{2}\right], \Gamma$ is the broaden- 

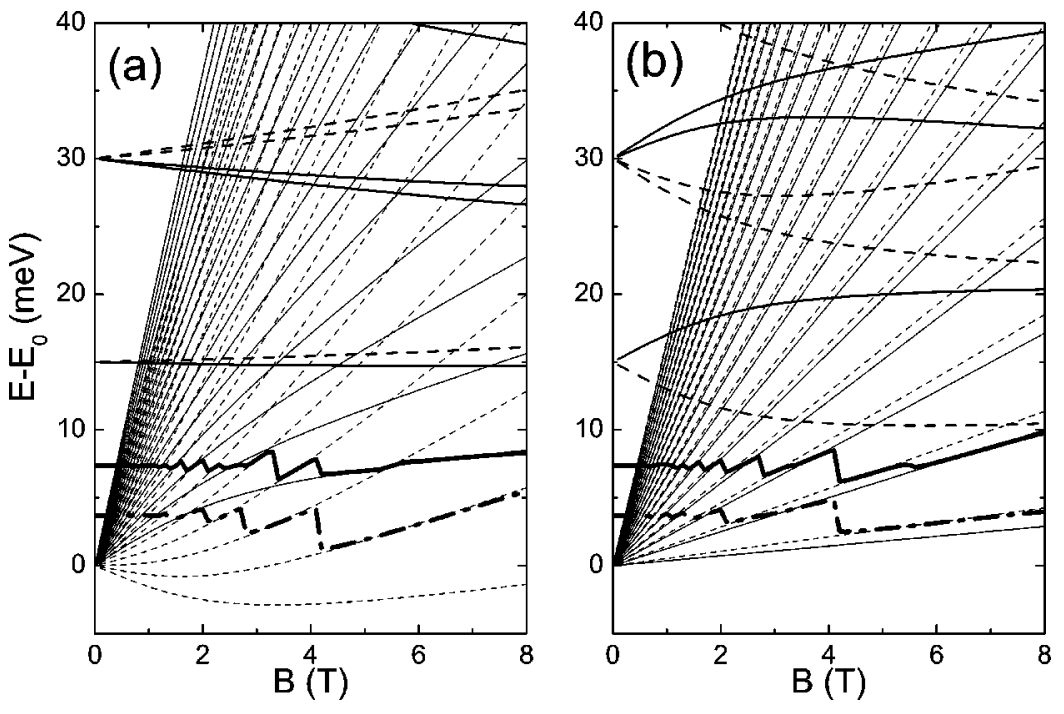

FIG. 2. The Landau-level fan in (a) DMS and (b) NMS 2DEG for spin-up (the thin solid lines) and spin-down (the thin dashed lines) electron. The thinner lines correspond to the energies of the spin-up (solid lines) and the spindown (dashed lines) electron states in the QD. The thickest curves depict the Fermi energy versus the magnetic fields, where the solid line and the dasheddotted line correspond to the electron density $\aleph_{e}=4 \times 10^{11} / \mathrm{cm}^{2}$ and $2 \times 10^{11} / \mathrm{cm}^{2}$, respectively. We set $T=1 \mathrm{~K}, \hbar \omega_{0}=15 \mathrm{meV}$. ing factor of the Landau level, and $V_{\text {ext }}$ is the external voltage between the emitter and the QD. The current matrix element in Eq. (10) is given by

$$
M_{e-d}=\left(\frac{\hbar^{2}}{2 m}\right) \int_{\Re}\left(\Psi_{e} \nabla \Psi_{d}^{*}-\Psi_{d}^{*} \nabla \Psi_{e}\right) d \Re=M_{e-d}^{\|} M_{e-d}^{\perp},
$$

where $\mathfrak{R}$ is the surface across the emitter-dot barrier, i.e., perpendicular to the $z$ axis. But here we calculate the transition element $M_{e-d}^{\perp}$ following Ref. 12, and consequently the in-plane transition element $M_{e-d}^{\|}$can be obtained analytically, ${ }^{13}$

$$
\begin{aligned}
M_{e-d}^{\|}= & \frac{1}{2 \pi} \int_{0}^{2 \pi} e^{i\left(m_{e}-m_{d}\right) \varphi} d \varphi \int_{0}^{\infty} r R_{n_{d}, m_{d}}(r) R_{n_{e}, m_{e}}(r) d r \\
= & \delta_{m_{d}, m_{e}} \frac{N_{d} N_{e}}{2} \Gamma(\gamma) \lambda^{-n_{d}-n_{e}-\gamma}\left(\lambda-k_{d}\right)^{n} d\left(\lambda-k_{e}\right)^{n_{e}} \\
& \times F\left[-n_{d},-n_{e}, \gamma, \frac{k_{d} k_{e}}{\left(\lambda-k_{d}\right)\left(\lambda-k_{e}\right)}\right],
\end{aligned}
$$

where $e$ and $d$ denote the states in the emitter and in the QD, $F(n, m, x, y)$ is the hypergeometric function, $\lambda=\left(a_{e}^{-2}\right.$ $\left.+a_{d}^{-2}\right) / 4, \quad k_{d, e}=1 /\left(2 a_{d, e}^{2}\right), \quad N_{d, e}$ $=\sqrt{\left(\left|m_{d, e}\right|+n_{d, e}\right) ! /\left(2^{\mid m_{d, e}}\left|n_{d, e} !\right| m_{d, e} \mid !\right)} / a_{d, e}^{\gamma}, \quad \gamma=\left|m_{d, e}\right|+1, \quad a_{d, e}$ $=\sqrt{\hbar /\left(2 m^{*} \omega_{d, e}\right)}$, and $\omega_{e}=w_{c} / 2$. It is interesting to notice that the angular part of the integral gives the selection rule for the tunneling process, i.e., only the states with $m_{e}=m_{d}$ contribute to the tunneling current.

The parameters used in our calculations for $\mathrm{Mn}_{x} \mathrm{Cd}_{1-x} \mathrm{Te}$ and CdTe are $m^{*}=0.096 m_{0}, \aleph_{e}=4 \times 10^{11} \mathrm{~cm}^{-2}, x=0.1, g_{\mathrm{Mn}}$ $=2, N_{0} \alpha=220 \mathrm{meV}, g_{e}=-1.47, S_{0}=1.32, T_{0}=3.1 \mathrm{~K}$ (Refs. 9,14) $\Gamma=0.2 \mathrm{meV}$.

\section{NUMERICAL RESULTS AND DISCUSSIONS}

Figure 1 schematically depicts our single barrier structure in which the QDs are embedded. The emitter and the collector regions consist of NMS or DMS 2DEG. Figures 2(a) and 2(b) show the energy spectra for the electron in the NMS and DMS 2DEG, respectively, as a function of the magnetic field for zero bias. The thickest curves denote the Fermi energy in the 2DEG for two different electron densities; the thick solid lines describe the energies of the electron states in (a) NMS and (b) DMS QD for different spin orientations. The Fermi energy exhibits the well known oscillatory behavior with an increasing magnetic field. The Landau-level fans for (a) NMS and (b) DMS 2DEG are rather different due to the $s-d$ exchange interaction. From these figures we see that the occupied states are spin-down states for DMS 2DEG at high magnetic fields, while spin-up and spin-down states coexist for NMS 2DEG, even at high magnetic fields. This feature arises from the s-d exchange interaction between the electron and the magnetic ions [see Eq. (3)]; namely, the spin splitting caused by the $s$ - $d$ exchange interaction is much larger than that caused by the intrinsic Zeeman effect. This feature consequently leads to a different spin polarization of the $2 \mathrm{DEG}$ at higher magnetic fields.

In Figs. 3(a) and 3(b) we plot the tunneling current due to the presence of the QD as a function of the magnetic field under a fixed bias for two different structures (DMS 2DEG contacts with NMS QD embedded in the barrier and NMS 2DEG contacts with DMS QD embedded in the barrier). The insets show the tunneling currents for the spin-up and the spin-down electrons. The tunneling current is mainly determined by the energy difference between the Landau levels in the emitter below the Fermi energy and the states in the QD and the overlap factor. The magnetic field shifts the minima of the subbands to higher energies and confines the electrons towards the center of the QD. The localization of the QD states leads to an increase of the overlap factor in the lateral direction, and consequently it increases the current. The tunneling current oscillates with an increasing magnetic field, but approaches zero at a high magnetic field because of the diminishing number of available tunnel channels. The oscillations in the current arise from those in the Landau levels below the Fermi energy, which become resonant with quantum states in the QD structure. In order to understand this more clearly, we plot the energy spectrum at a fixed bias $V_{\text {ext }}=10 \mathrm{mV}$ in Figs. 3(c) and 3(d). From Figs. 3(c) and 3(d), 

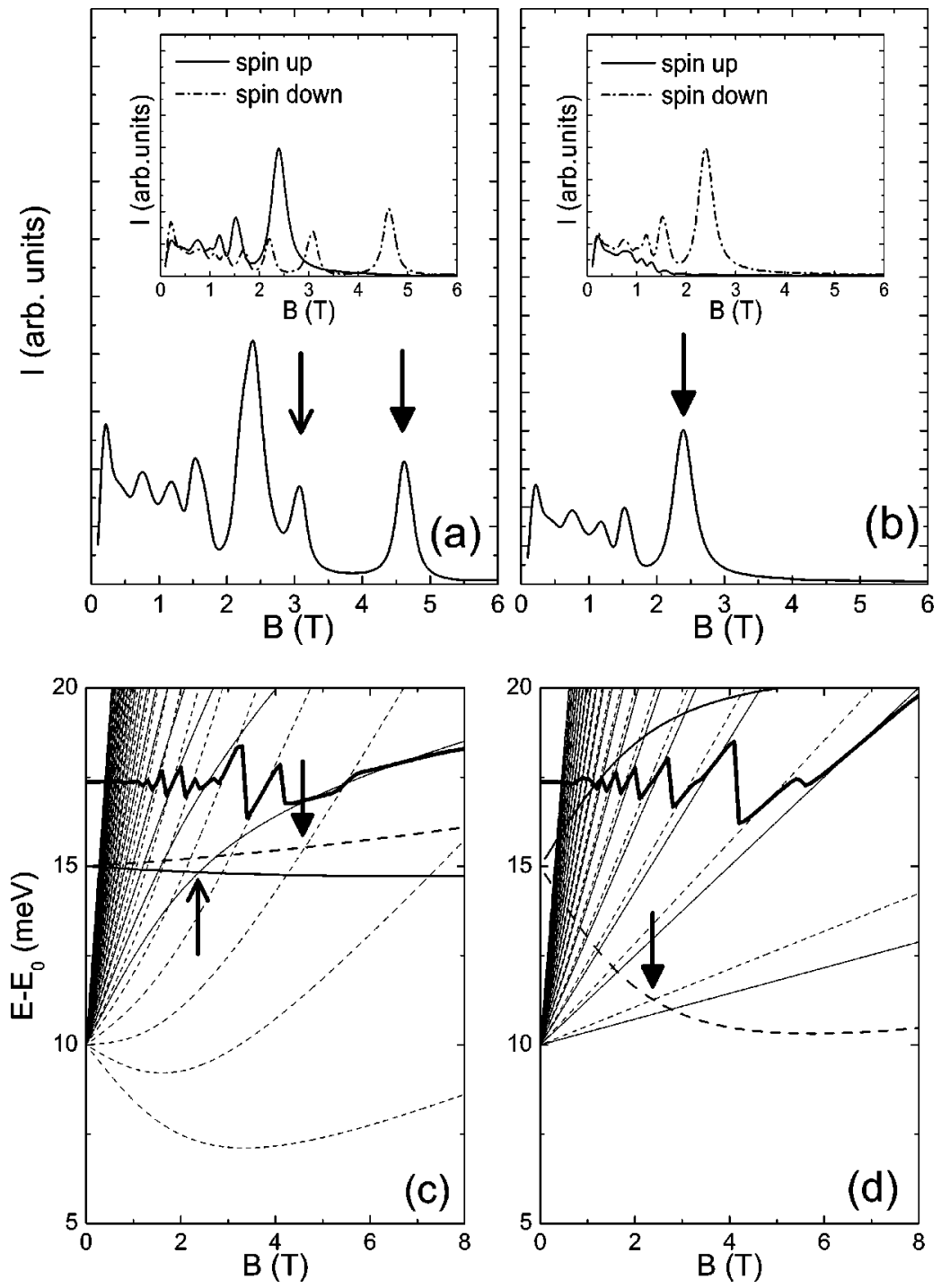

FIG. 3. The tunneling current versus the magnetic field for a fixed bias $(\Delta \mathrm{V}=10 \mathrm{mV})$ for the (a) NMS/DMS QD/NMS and (b) DMS/NMS QD/DMS cases. The insets show the tunneling current for different spin orientations. Parts (c) and (d) show the corresponding energy spectra in the emitter and the QD. The thick solid lines denote the Fermi energy in the emitter, the thin solid and dashed lines for the spin-up and the spin-down Landau levels in the emitter and the thicker solid and dashed lines for the spin-up and the spin-down QD states. We set $\hbar \omega_{d}=15 \mathrm{meV}$, $\Gamma=0.2 \mathrm{meV}$, and $T=1 \mathrm{~K}$. the spin-up and the spin-down Landau levels in the emitter cross with the spin-up and the spin-down ground states of the QD at different magnetic fields. These crossing points correspond to the peak positions in Figs. 3(a) and 3(b) (see the arrows in these figures). The minimum of the lowest Landau subband of the DMS 2DEG increases with an increasing magnetic field and even becomes higher than that of the ground state of the QD $\left(m_{d}=0\right)$, but lower than that of the higher states of the QD $\left(m_{d}=1\right)$ [see Figs. 2(a) and 2(b)]; therefore the tunneling current approaches zero at a higher magnetic field. The same is true for the NMS/DMS QD/ NMS structure due to the same physical mechanism. A big difference between the spin-up and spin-down tunneling currents is found in these figures, even at intermediate magnetic fields [see the insets of Figs. 3(a) and 3(b)], resulting in a spin polarization of the current. For tunneling through a NMS QD with DMS contacts, the spin polarization of the tunneling current is higher than the spin polarization of the tunneling current through the DMS QD with NMS contacts. This is because the lower states of the DMS 2DEG are spindown states, but the spin-up states are also still present in the NMS 2DEG [see Figs. 2(a) and 2(b)].
Figures 4(a)-4(d) show the tunneling current versus the external bias for two different values of the magnetic fields. The insets depict the tunneling currents for the spin-up and the spin-down electrons separately. We find several broad peaks in the tunneling current, and the spin polarization can be easily tuned by changing the external bias, even at small magnetic fields (see the insets). These broad peaks correspond to the Landau levels below the Fermi energy in the 2DEG, which are resonant with the QD states (the ground and excited states) and are indicated in Figs. 4(e) and 4(f). The minima of the tunneling current correspond to the Landau levels below the Fermi energy, which are situated between the energies of the QD states $\left(m_{d}=0\right.$ and $\left.m_{d}=1\right)$, and the widths of the minima are determined by the spacing of the QD states. Notice that there are many subpeaks, i.e., oscillation, on top of each broad peak for sufficiently strong magnetic fields. These sharp subpeaks appear when each Landau level becomes resonant with the QD states and appears only if the Landau-level separation becomes larger than the width of the individual Landau levels. For tunneling through the NMS QD with DMS contacts [see Figs. 4(a) and 4(b)], a maximum of spin polarization can be up to $35 \%$ and $90 \%$ for $B=0.2 \mathrm{~T}$ and $0.8 \mathrm{~T}$ respectively. Except for the tun- 

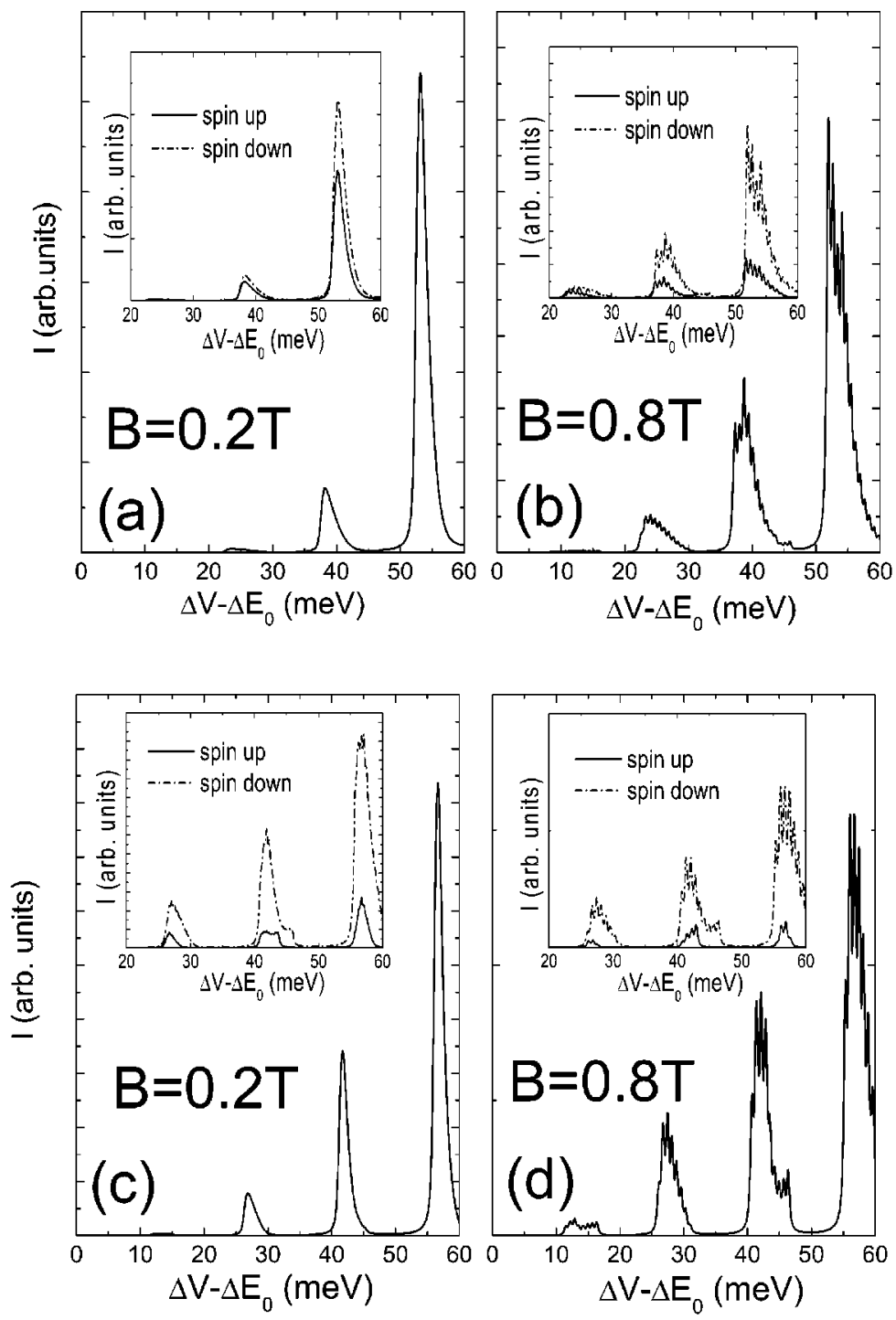

FIG. 4. The tunneling current versus the external bias for two different magnetic field values and two different configuratons: NMS/DMS QD/ NMS [(a) and (b)] and DMS/NMS QD/DMS [(c) and (d)]. The insets show the tunneling current for different spin orientations. The energy spectra are shown as a function of the external bias in (e) and (f), and the line types are the same as in Figs. $3(\mathrm{c})$ and 3(d). We used $\hbar \omega_{0}=15 \mathrm{meV}, \Gamma$ $=0.2 \mathrm{meV}, T=1 \mathrm{~K}$, and $\Delta E_{0}=E_{0}^{d}-E_{0}^{e}$, where $E_{0}^{d, e}$ are the quantized energies along the $z$ axis of the QD and the 2DEG.
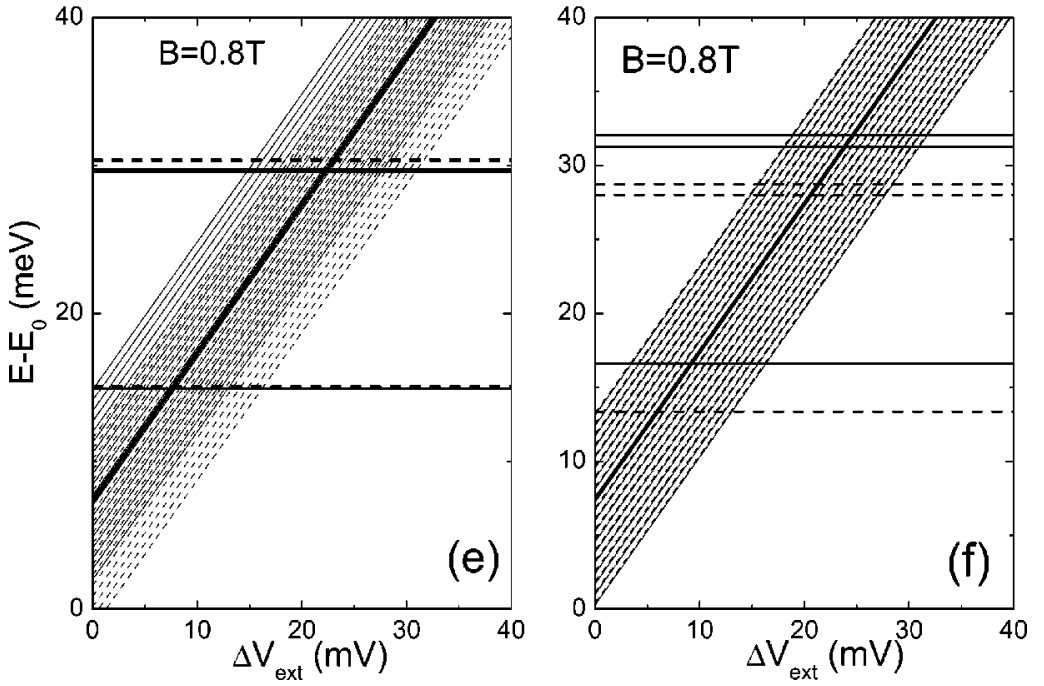

neling through the DMS QD with NMS contacts [see Figs. 4(c) and 4(d)], the maximum of the spin polarization can approach $100 \%$, even at small magnetic fields (i.e., $B$ $=0.2 \mathrm{~T}$ ). Therefore, spin polarization of the tunneling current through the NMS QD with DMS contacts is stronger than that through the DMS QD with NMS contacts. This phenomenon can be understood from the energy spectra of the NMS and the DMS 2DEG for a fixed magnetic field $(B=0.8 \mathrm{~T})$ 
[see Figs. 4(e) and 4(f)]. The QD states align with the lower Landau levels of the 2DEG with an increasing external bias. The lower Landau levels of the DMS (NMS) 2DEG [see Figs. 4(e) and 4(f)] are the spin-down (the spin-up and the spin-down) states. Therefore, the spin polarization of the tunneling current through the NMS QD with the DMS 2DEG contacts is higher than that through the DMS QD with the NMS 2DEG contacts.

\section{CONCLUSIONS}

In summary, we demonstrated theoretically that strong spin-polarized current can be realized by utilizing the tunneling between a 2DEG and a QD structure even at small mag- netic fields. The tunneling current oscillates and decreases with an increasing magnetic field. The spin polarization of the system can be easily tuned by changing the external bias under a weak magnetic field. The proposed system is useful as, e.g., a spin filter.

\section{ACKNOWLEDGMENTS}

This work is partly supported by the NSFC No. (Grant No.) 60376016,863 project 2003AA311070, the special fund for Major State Basic Research Project No. (Grant No.) G001CB3095 of China, and HKRGC, Project No. (Grant No.) CityU 100303, the Flemish Science Foundation (FWOVI), Belgian Science Policy and the Bilateral Cooperation programme between Flanders and China.
*Email address: kchang@red.semi.ac.cn

${ }^{1}$ G. A. Prinz, Phys. Today 48 (4), 58 (1995); Science 282, 1660 (1998), and references therein.

${ }^{2}$ J. C. Egues, Phys. Rev. Lett. 80, 4578 (1998).

${ }^{3}$ R. Fiederling, M. Keim, G. Reuscher, W. Ossau, G. Schmidt, A. Waag, and L. W. Molenkamp, Nature (London) 402, 787 (1999); Y. Ohno, D. K. Young, B. Benschoten, F. Matsukura, H. Ohno, and D. D. Awschalom, Nature (London) 402, 790 (1999).

${ }^{4}$ J. C. Egues, Phys. Rev. B 64, 195319 (2001); Kai Chang, J. B. Xia, and F. M. Peeters, ibid. 65, 115209 (2002).

${ }^{5}$ Kai Chang, J. B. Xia, and F. M. Peeters, Phys. Rev. B 65, 155211 (2002).

${ }^{6}$ S. H. Xin, P. D. Wang, A. Yin, C. Kim, M. Dobrowolska, J. L. Merz, and J. K. Furdyna, Appl. Phys. Lett. 69, 2884 (1996); Y. Oka, J. Shen, K. Takabayashi, N. Takahashi, H. Mitsu, I. Souma, and R. Pittini, J. Lumin. 83, 83 (1999); C. S. Kim, M. Kim, S. Lee, J. Kossut, J. K. Furdyna, and M. Dobrowolska, J. Cryst. Growth 214, 395 (2000).

${ }^{7}$ A. A. Maksimov, G. Bacher, A. MacDonald, V. D. Kulakovskii, A. Forchel, C. R. Becker, G. Landwehr, and L. W. Molenkamp,
Phys. Rev. B 62, R7767 (2000); G. Bacher, H. Schomig, M. K. Welsch, S. Zaitsev, V. D. Kulakovskii, A. Forchel, S. Lee, M. Dobrowolska, J. K. Furdyna, B. Konig, and W. Ossau, Appl. Phys. Lett. 79, 524 (2001).

${ }^{8}$ J. Kossut, I. Yamakawa, A. Nakamura, G. Cywiński, K. Fronc, M. Czeczott, J. Wróbel, F. Kyrychenko, T. Wojtowicz, and S. Takeyama, Appl. Phys. Lett. 79, 1789 (2001).

${ }^{9}$ Kai Chang and J. B. Xia, J. Phys.: Condens. Matter 14, 13661 (2002); Kai Chang, J. B. Xia, and F. M. Peeters, Appl. Phys. Lett. 82, 2661 (2003).

${ }^{10}$ J. Bardeen, Phys. Rev. Lett. 6, 57 (1961).

${ }^{11}$ M. Wagner, Phys. Rev. Lett. 85, 174 (2000).

${ }^{12}$ S. L. Chuang and N. Holonyak, Jr., Appl. Phys. Lett. 80, 1270 (2002).

${ }^{13}$ L. Landau and E. Lifshitz, Quantum Mechanics (Pergamon Press, New York, 1977).

${ }^{14}$ Physics of II-VI and I-VII Compounds, Semimagnetic Semiconductors, edited by O. Madelung, M. Schulz, and H. Weiss, Landolt-Börnstein, New Series, Group III, Vol. 17, Pt. B (Springer, Berlin, 1982). 